\title{
Periodic Behaviour of Mean Velocity Fields in Rushton Turbine (RT) Driven Stirred Tank
}

\author{
Devarajan Krishna Iyer \\ Department of Civil Engineering, \\ National Institute of Technology Warangal, \\ Warangal-506004, Telangana, India \\ E-mail: kdevarajan92@gmail.com \\ Ajey Kumar Patel \\ Department of Civil Engineering, \\ National Institute of Technology Warangal, \\ Warangal-506004, Telangana, India \\ Corresponding author: ajeypatel@gmail.com
}

(Received December 20, 2018; Accepted August 17, 2019)

\begin{abstract}
The present study predicts the periodic behavior of mean velocity fields from the properly verified and validated CFD model to determine the extent of vortex and turbulent activity in a baffled tank stirred by standard six bladed Rushton Turbine. This region includes most of the vortex and turbulent action that controls the mixing and mass transfer processes in the stirred tank. The complexity of periodicity of mean radial and tangential velocities in the radial direction and mean axial velocity in the axial direction is used to fix the proper radial as well as axial extents of the vortex generated by RT. The extent of flow periodicity in the present stirred tank configuration is confined within a cylindrical region around the impeller of radius 0.753 times the impeller diameter and a height of 0.323 times the impeller diameter above and below the impeller center plane.
\end{abstract}

Keywords- CFD, Turbulent flow, Baffled stirred tank, Periodicity.

\section{Introduction}

Stirred tanks are generally used for mixing and dispersion activities in various industrial processes (Basavarajappa et al., 2015). The Computational Fluid Dynamics (CFD) provides a broad insight in to the flow fields and alleviates various difficulties linked with experimental studies to achieve the efficient design of the stirred tank reactors (Coroneo et al., 2011). The steady state Multiple Reference Frame (MRF) impeller modelling scheme with standard $k-\varepsilon$ turbulence model provide economical as well as promising flow field predictions if the errors arising from grid resolution and discretization scheme are suitably minimized (Wechsler et al., 1999; Aubin et al., 2004; Deglon and Meyer, 2006; Coroneo et al., 2011). But this effective model combination isn't properly used for quantitative analysis of periodic mean velocity fluctuations as well as the extent of vortex and turbulent action present in the agitated vessels. Further, the CFD model provides the details of mean velocities within the blades which can't be achieved from the sophisticated experimental methods (Alcamo et al., 2005). Thus the main objective of the present work is to use the verified and validated CFD model for achieving detailed insight in to the periodic nature of mean velocity fields within the blades as well as in the impeller discharge stream in order to estimate the extent of vortex and turbulent action in a baffled tank stirred by standard six bladed RT. 
International Journal of Mathematical, Engineering and Management Sciences

Vol. 4, No. 6, 1341-1351, 2019

https://dx.doi.org/10.33889/IJMEMS.2019.4.6-105

\section{Stirred Tank Configuration}

The stirred tank used for the numerical experiments confirms to a standard configuration which was used by $\mathrm{Wu}$ and Patterson (1989) for analyzing the turbulent flow characteristics. The geometry consists of a flat bottomed completely baffled tank having an equal diameter $(T)$ and height $(H)$. The tank was completely filled with water at standard conditions (Temperature of $25^{\circ} \mathrm{C}$, Density of $998 \mathrm{Kg} / \mathrm{m}^{3}$ and viscosity of $0.001 \mathrm{Kg} / \mathrm{ms}$ ). The standard six bladed RT with the diameter $(D)$ of $T / 3$ placed at $H / 3$ from the tank bottom was used for mixing the fluid. The impeller was rotated at a speed of $200 \mathrm{rpm}$ to generate the turbulent conditions corresponding to a Reynolds number of 28,830 . The sectional elevation and plan of the stirred tank are shown in Figure 1.



(a)

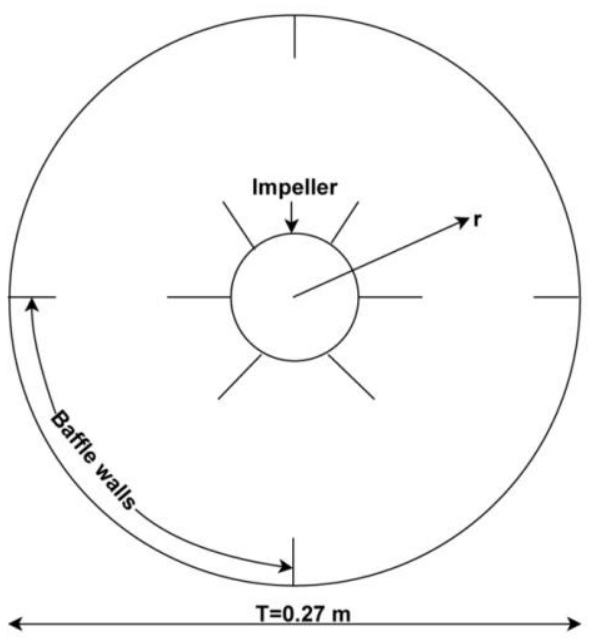

(b)

Figure 1. (a) Sectional elevation and (b) Plan of baffled stirred tank (Wu and Patterson, 1989) 
International Journal of Mathematical, Engineering and Management Sciences

Vol. 4, No. 6, 1341-1351, 2019

https://dx.doi.org/10.33889/IJMEMS.2019.4.6-105

\section{Computational Methodology}

The steady state three dimensional CFD models were developed using commercially available finite volume based ANSYS 17.0 software. The flow domain was discretized using tetrahedral elements in the rotating zone containing impeller and using hexahedral elements in the stationary zone containing baffle walls as shown in Figure 2.

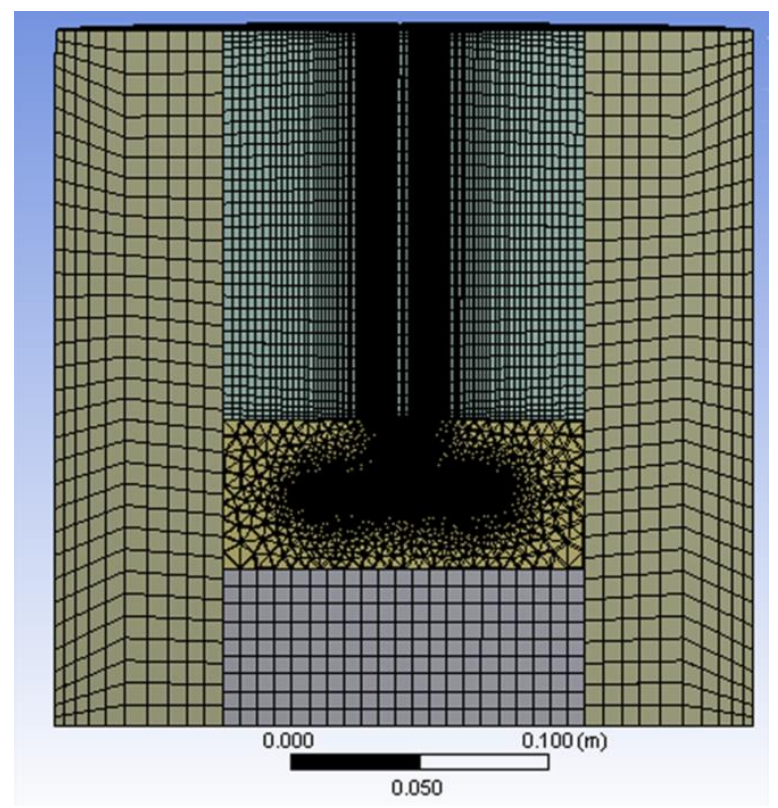

(a)



(b)

Figure 2. (a) Sectional elevation (b) Plan of meshed geometry 
International Journal of Mathematical, Engineering and Management Sciences

Vol. 4, No. 6, 1341-1351, 2019

https://dx.doi.org/10.33889/IJMEMS.2019.4.6-105

The flow variables such as pressure, velocity and turbulent quantities were computed using RANS approach with standard $k-\varepsilon$ turbulence model closure. The standard coefficients of the turbulent viscosity recommended by ANSYS (2013) were retained in the respective model expressions. The impeller disc surfaces, blades, baffles and tank periphery were modelled as walls with no-slip boundary condition while tank top was treated using symmetry boundary condition. The impeller-baffle interactions were modelled using the MRF method and the resulting equations were spatially discretized using higher order discretization schemes. The discretized equations were coupled using SIMPLE pressure-velocity coupling scheme and the calculations were performed by right monitoring of impeller torque, volume integral of turbulent dissipation rate and residuals of all the flow variables respectively. The computations were considered as converged when the residuals of all the flow variables fall below $10^{-6}$. A workstation having 64 bit Intel (R) Xeon (R) E5-1620 3.6 GHz processor was used for the simulations.

\section{Results and Discussion}

The preliminary phase of the work is to conduct simulations at various grid resolutions and discretization schemes to minimize the numerical errors present in the flow field results and to validate the same by comparing with the experimental results available in the literature of $\mathrm{Wu}$ and Patterson (1989) and Bates et al. (1963). The next phase is to analyze the extent of the vortex and turbulent activity around the impeller from the predicted mean velocity fields.

\subsection{Verification and Validation of Flow Field Results}

The variation of power number from torque $\left(N_{p}\right)$ and axial profile of normalized radial velocity $\left(U_{r} / U_{t i p}\right)$ close to the impeller tip with grid resolution and discretization schemes are shown in Figures 3 and 4 respectively.

The grid independence study was conducted at three different grid resolutions with a constant refinement factor of 1.48 and using second order upwind discretization scheme. The resulting optimal grid was used to study the effects of first order upwind as well as QUICK discretization schemes.

The grid resolution and discretization schemes have a huge impact on $N_{p}$ as well as the axial profile of $U_{r} / U_{t i p}$ respectively. The discretization error in $N_{p}$ represented as the Grid Convergence Index (GCI) (Roache, 1994) reduces from 2.8\% in the medium grid to $1.5 \%$ in the fine grid indicating minor differences in the results between medium and fine grids respectively. The axial profile of $U_{r} / U_{\text {tip }}$ predicted by medium and fine grids are highly comparable as the GCI of peak velocity in the fine mesh is $5 \%$. But the GCI of peak velocity in medium mesh increases to $33.8 \%$ as the axial profile of $U_{r} / U_{\text {tip }}$ from coarse mesh has much deviated from the medium mesh. Thus the fine mesh having lesser GCI of flow field results was used for the further analysis. 
International Journal of Mathematical, Engineering and Management Sciences

Vol. 4, No. 6, 1341-1351, 2019

https://dx.doi.org/10.33889/IJMEMS.2019.4.6-105

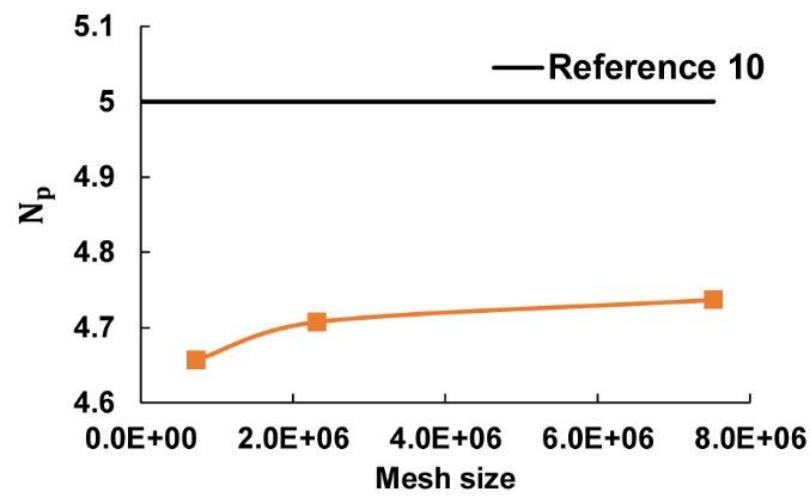

(a)

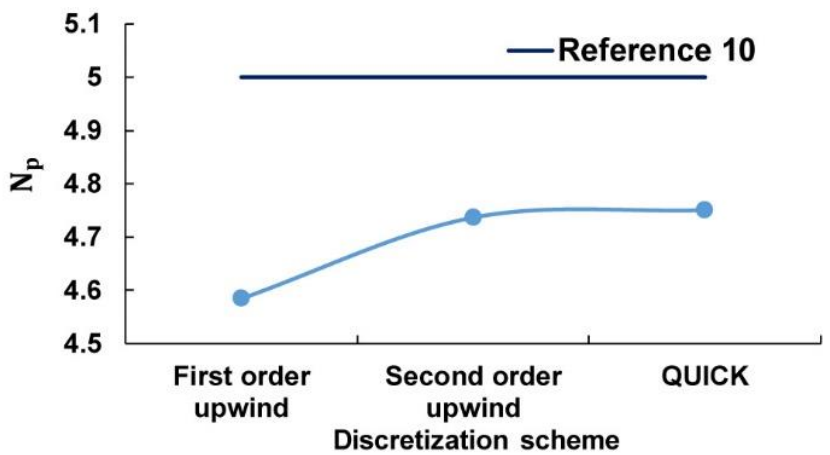

(b)

Figure 3. Effects of (a) grid resolution and (b) discretization schemes on $N_{p}$



Figure 4. Effects of grid resolution and discretization schemes on axial profile of $U_{r} / U_{t i p}$ close to the impeller tip 
International Journal of Mathematical, Engineering and Management Sciences

Vol. 4, No. 6, 1341-1351, 2019

https://dx.doi.org/10.33889/IJMEMS.2019.4.6-105

The predictions of $N_{p}$ as well as the axial profile of $U_{r} / U_{\text {tip }}$ from higher order discretization schemes (second order upwind and QUICK) were almost same while the first order upwind scheme under predicts the respective flow variables as evident from Figures 3(b) and 4 respectively. The relative variation of $3.2 \%$ as well as $4.6 \%$ can be seen in the $N_{p}$ and peak radial velocity predictions as the discretization scheme changes from first order upwind to second order upwind scheme. Thus the fine mesh discretized using second order upwind scheme was used for the validation as well as the analysis purposes.

The $N_{p}$ from the completely verified model is close to the experimental results of Bates et al. (1963) as the percentage deviation in the same is limited to $5 \%$. The axial profile of $U_{r} / U_{t i p}$ is comparable with the experimental findings of $\mathrm{Wu}$ and Patterson (1989) as the mean absolute error is around 0.06 and percentage deviation in the peak velocities is limited to $1.2 \%$. Thus the fine mesh discretized using second order upwind scheme reproduces the flow quantities that are close to the standard results and can be used for further analysis and interpretations.

\subsection{Extent of Trailing Vortex and Turbulent Action around the RT Impeller}

The periodic variation of normalized mean radial $\left(U_{r} / U_{t i p}\right)$, tangential $\left(U_{t} / U_{t i p}\right)$ and axial $\left(U_{a} / U_{t i p}\right)$ velocities behind the blade at various radial distances from the impeller centre as well as at various axial planes above and below the impeller center plane were analyzed. The radial distances were normalized with the impeller radius $(R)$ and represented as $r^{*}$ while the axial locations were normalized with half blade width $\left(\frac{w}{2}\right)$ and indicated as $z^{*}$. The inferences regarding the angular variation of velocities were drawn at different regions viz. (a) within the impeller blades $\left(r^{*}\right.$ between 0.753 and 0.968$)$, (b) impeller discharge stream $\left(r^{*}\right.$ between 1.022 and 2.15), (c) within the axial extent of impeller blades $\left(z^{*}\right.$ of \pm 0.86$)$ and (d) outside the axial extent of impeller blades $\left(z^{*}\right.$ of \pm 2.58$)$. The \pm sign indicates the $z^{*}$ above and below the impeller center plane. The Figures 5-7 show the periodic variation of mean radial and tangential velocities in the impeller center plane $\left(z^{*}=0\right)$ as well as the axial velocity at the $z^{*}$ of -0.86 .

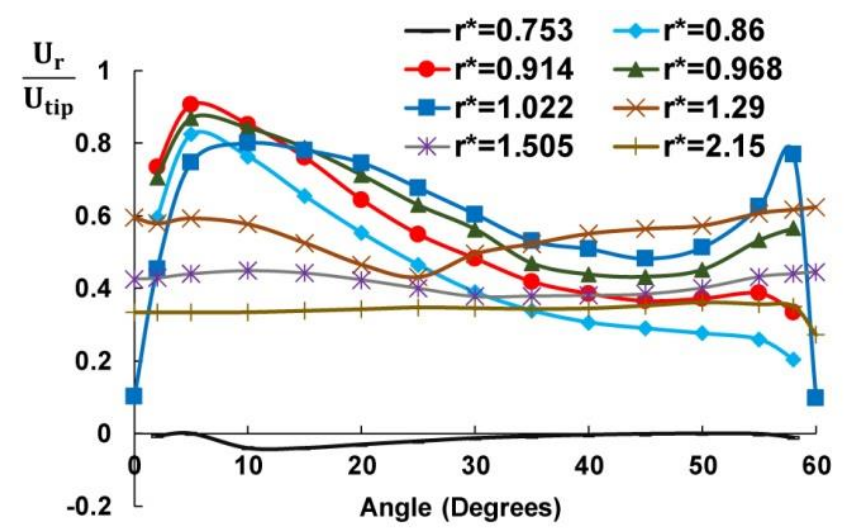

Figure 5. Periodic variation of $U_{r} / U_{\text {tip }}$ at $z^{*}=0$ 
International Journal of Mathematical, Engineering and Management Sciences

Vol. 4, No. 6, 1341-1351, 2019

https://dx.doi.org/10.33889/IJMEMS.2019.4.6-105

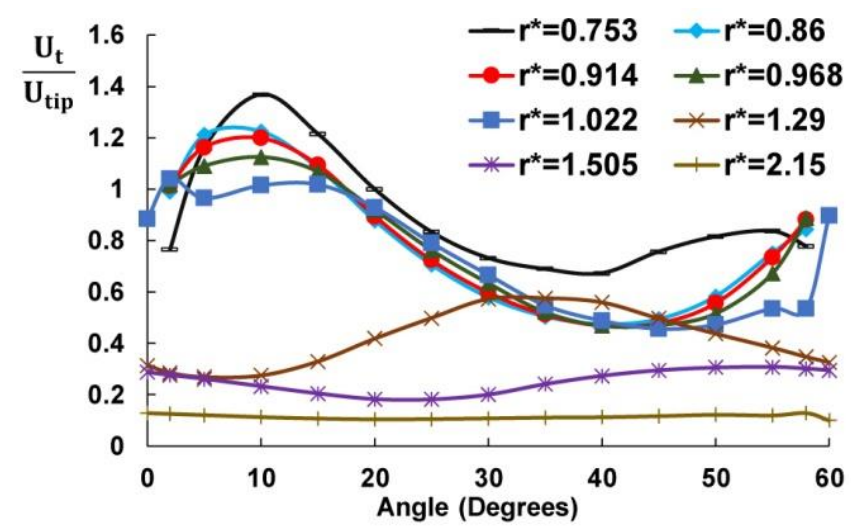

Figure 6. Periodic variation of $U_{t} / U_{t i p}$ at $z^{*}=0$

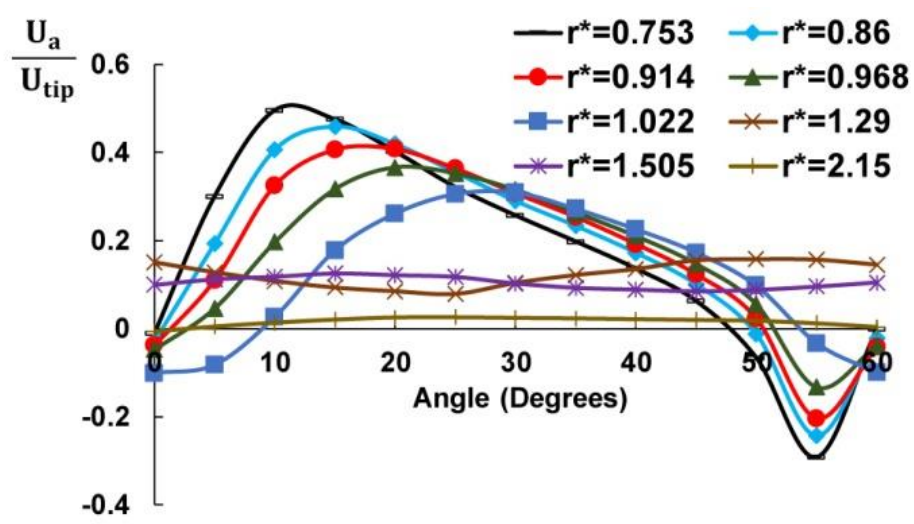

Figure 7. Periodic variation of $U_{a} / U_{\text {tip }}$ at $z^{*}=-0.86$

Apart from the periodic behavior of mean velocities, the basic flow dynamics of RT driven stirred tanks can also be explained using these figures. The zero degree in the Figures 5-7 corresponds to the center of leading blade while sixty degree corresponds to the center of the successive blade. The basic hydrodynamics of stirred tank reactors include the dominance of tangential velocity within the blades and faster decay of the same in the impeller discharge stream as compared to the radial velocity. The peak tangential velocity of magnitude $1.37 U_{t i p}$ was found at $10^{\circ}$ behind the blades while the peak radial velocity of magnitude $0.907 U_{\text {tip }}$ occurs at $5^{\circ}$ behind the blades. The peak axial velocity is comparatively lesser than the other velocities and found to be $0.496 U_{\text {tip }}$ at $10^{\circ}$ behind the blades. Further, the peak mean velocities behind the impeller blades provide clear evidence of vortex action and the same shifts radially as well as azimuthally away from the impeller.

The comprehensive mean velocity details extracted from the present CFD model exhibit strongest periodicity in the radial as well as tangential velocities along the impeller center plane which decreases with the axial distance above and below the impeller center plane. The sinusoidal 
International Journal of Mathematical, Engineering and Management Sciences

Vol. 4, No. 6, 1341-1351, 2019

https://dx.doi.org/10.33889/IJMEMS.2019.4.6-105

pattern at various $r^{*}$ in the Figures 5-7 represent periodic nature of mean velocities while the flat profiles indicate the non-periodic nature of the same. The periodic nature of mean velocities clearly depicts the presence of trailing vortices and the extent of periodicity indicates the active region of the vortex and turbulent action around the RT impeller (Stoots and Calabrese, 1995; Rutherford et al., 1996). Along the axial extent of the impeller blade, strong periodicity in the mean radial and tangential velocities can be observed within the impeller blades and corresponding decay in the impeller discharge region. The periodicity in the mean radial velocity is absent at the $r^{*}$ of 0.753 and increases up to the $r^{*}$ of 0.914 followed by the continuous decrease of the same until the $r^{*}$ of 1.4. But mean tangential velocity shows strongest periodicity at the $r^{*}$ of 0.753 and maintains the same within the impeller blades followed by faster decay until the $r^{*}$ of 1.29. Beyond the axial extent of the impeller blades, periodicity in the mean tangential velocity continuously reduces from the $r^{*}$ of 0.753 to 1.4 while the periodic nature of mean radial velocity increases from the $r^{*}$ of 0.753 to 1.075 followed by continuous decrease of the same until the $r^{*}$ of 1.4. The periodic nature of mean radial and tangential velocities at the $z^{*}$ of \pm 1.72 are shown in Figures 8-9 respectively.

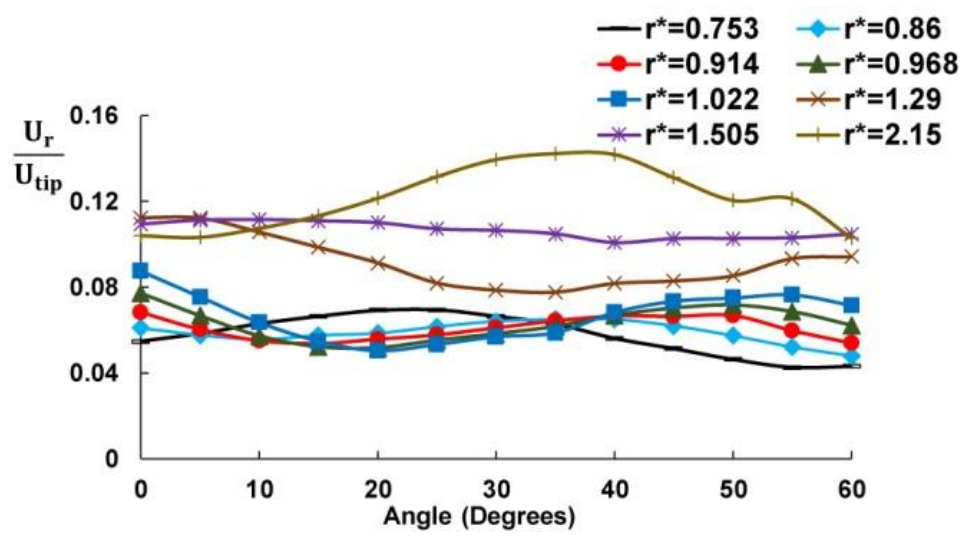

Figure 8. Periodic variation of $U_{r} / U_{\text {tip }}$ at $z^{*}=-1.72$



Figure 9. Periodic variation of $U_{t} / U_{\text {tip }}$ at $z^{*}=1.72$ 
International Journal of Mathematical, Engineering and Management Sciences

Vol. 4, No. 6, 1341-1351, 2019

https://dx.doi.org/10.33889/IJMEMS.2019.4.6-105

Finally, the mean radial and tangential velocities become completely non-periodic at the $r^{*}$ of 1.505 and $z^{*}$ of \pm 2.58 . The higher magnitude and periodicity of mean tangential velocity within the impeller blades leads to swirling motion of the radial jet emerging behind the blades while the higher magnitude and periodicity of radial velocity in the impeller stream constitutes strong radial discharge from the impeller. The higher magnitude of tangential velocity within the blades as compared to the radial as well as impeller tip velocities is due to the acceleration of the fluid over the blades (Stoots and Calabrese, 1995) and decays much faster than mean radial velocity in the impeller discharge stream. Moreover, the mean radial velocity predominates the axial and tangential velocities outside the axial extent of the impeller blades. These facts confirm the strong radial pumping action beyond the axial and radial extents of the RT impeller.

The periodicity in the mean axial velocity is quite less in the impeller center plane and increases above and below the impeller center plane up to the $z^{*}$ of \pm 0.86 and decreases further until the $z^{*}$ of \pm 2.58 . Thus the strong periodic nature of mean axial velocity is present within the blade height and weakens outside the axial extent of the impeller blades. The strong periodic behavior of mean axial velocity above and below the impeller center plane was obtained at the $r^{*}$ between 0.753 and 0.86 followed by continuous decrease of the same in the impeller discharge region up to the $r^{*}$ of 1.4. Finally, mean axial velocity becomes non-periodic at the $r^{*}$ of 1.505 and $z^{*}$ of \pm 2.58 as shown in Figures 10-11.

The peak mean axial velocity is located above and below the impeller center plane as compared to impeller centerline location of peak radial and tangential velocities. The fluctuations in all the mean velocity patterns at the $r^{*}$ between 1.72 and 2.15 for the $z^{*}$ between \pm 1.29 and \pm 2.15 is attributed due to the shedding of vortices behind the baffle walls.

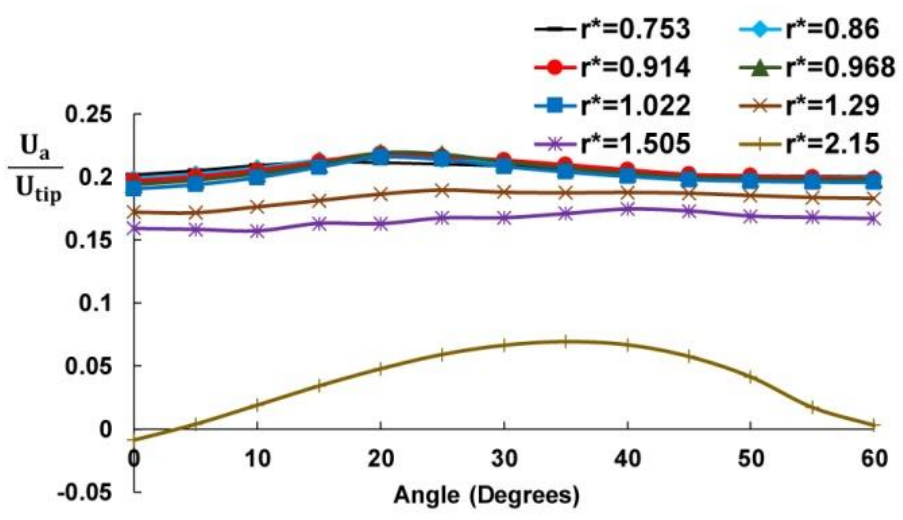

Figure 10. Periodic variation of $U_{a} / U_{t i p}$ at $z^{*}=-2.58$ 
International Journal of Mathematical, Engineering and Management Sciences

Vol. 4, No. 6, 1341-1351, 2019

https://dx.doi.org/10.33889/IJMEMS.2019.4.6-105

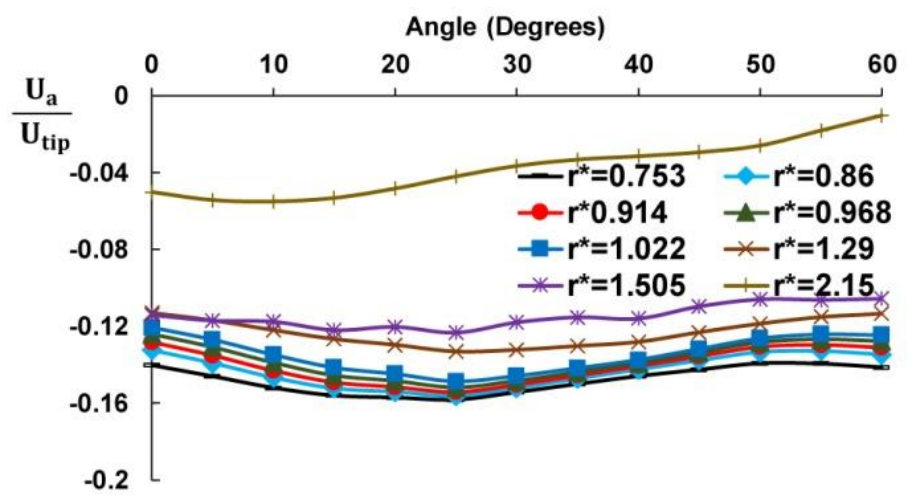

Figure 11. Periodic variation of $U_{a} / U_{\text {tip }}$ at $z^{*}=2.58$

The analysis reveals strong angular dependence of the mean velocities within the impeller blades and decay of the same in the impeller discharge region further. The radial and tangential velocities show strong periodic behavior in the radial direction while the corresponding variations in the axial direction are quite less outside the impeller blade height. But the axial velocity shows strong periodicity in the axial direction while the corresponding pattern in the radial direction remains unchanged. Thus angular variations in the radial and tangential velocities can be used to fix the radial extent of flow periodicity while the angular variations in the axial velocity can be used to estimate the axial extent of flow periodicity. The flow periodicity in the present configuration is confined within a cylindrical region around the impeller having a radius of 0.753 times the impeller diameter and height of 0.323 times the impeller diameter above and below the impeller center plane. The trailing vortices and major turbulent activity are mainly concentrated within this cylindrical region and doesn't have much effects outside of it. The extent of flow periodicity obtained from this study is not much deviated from the results of Stoots and Calabrese (1995) even though sufficient difference exists in the impeller clearances adopted in both the studies.

\section{Conclusion}

The extent of the vortex and turbulent action in a standard baffled tank stirred by six bladed RT was investigated using the completely verified and validated CFD model. The detailed investigation of mean velocity fields exhibit strong periodic behavior within the extents of the impeller blades and subsequently weaken radially as well as axially outside the impeller. The extent of flow periodicity in the radial direction can be determined from the radial and tangential velocity patterns while that in the axial direction can be obtained from axial velocity patterns around the impeller blades. The flow periodicity in the respective stirred tank is confined within a cylindrical region around the impeller having a radius of 0.753 times the impeller diameter and height of 0.323 times the impeller diameter above and below the impeller center plane. The extent of the periodic behavior of mean velocities represents the region of vigorous mixing due to the presence of high levels of vortex and turbulent structures which helps in designing the new impellers as well as finding appropriate locations for feeding the chemical reactants. 
International Journal of Mathematical, Engineering and Management Sciences

Vol. 4, No. 6, 1341-1351, 2019

https://dx.doi.org/10.33889/IJMEMS.2019.4.6-105

\section{Conflict of Interests}

Authors confirm that the present journal article contents have no conflict of interest.

\section{Acknowledgements}

Financial support from the Science and Engineering Research Board (SERB) of Government of India under the research project, Reference number SB/S3/CEE/0057/2013, is gratefully acknowledged.

\section{References}

Alcamo, R., Micale, G., Grisafi, F., Brucato, A., \& Ciofalo, M. (2005). Large-eddy simulation of turbulent flow in an unbaffled stirred tank driven by a Rushton turbine. Chemical Engineering Science, 60(8-9), 2303-2316.

ANSYS Inc. (2013). ANSYS fluent theory guide. Release 15.0, ANSYS, USA.

Aubin, J., Fletcher, D.F., \& Xuereb, C. (2004). Modeling turbulent flow in stirred tanks with CFD: the influence of the modeling approach, turbulence model and numerical scheme. Experimental Thermal and Fluid Science, 28(5), 431-445.

Basavarajappa, M., Draper, T., Toth, P., Ring, T.A., \& Miskovic, S. (2015). Numerical and experimental investigation of single phase flow characteristics in stirred tanks using Rushton turbine and flotation impeller. Minerals Enginering, 83, 156-167.

Bates, R.L., Fondy, P.L., \& Corpstein, R.R. (1963). An examination of some geometric parameters of impeller power. Industrial \& Engineering Chemistry Process Design and Development, 2(4), 310-314.

Coroneo, M., Montante, G., Paglianti, A., \& Magelli, F. (2011). CFD prediction of fluid flow and mixing in stirred tanks: Numerical issues about the RANS simulations. Computers \& Chemical Engineering, 35(10), 1959-1968.

Deglon, D.A., \& Meyer, C.J. (2006). CFD modelling of stirred tanks: Numerical considerations. Minerals Engineering, 19(10), 1059-1068.

Roache, P.J. (1994). Perspective: a method for uniform reporting of grid refinement studies. Journal of Fluids Engineering, 116(3), 405-413.

Rutherford, K., Lee, K.C., Mahmoudi, S.M.S., \& Yianneskis, M. (1996). Hydrodynamic characteristics of dual Rushton impeller stirred vessels. AIChE Journal, 42(2), 332-346.

Stoots, C.M., \& Calabrese, R.V. (1995). Mean velocity field relative to a Rushton turbine blade. AIChE Journal, 41(1), 1-11.

Wechsler, K., Breuer, M., \& Durst, F. (1999). Steady and unsteady computations of turbulent flows induced by a $4 / 45^{\circ}$ pitched-blade impeller. Journal of Fluids Engineering, 121(2), 318-329.

Wu, H., \& Patterson, G.K. (1989). Laser-Doppler measurements of turbulent-flow parameters in a stirred mixer. Chemical Engineering Science, 44(10), 2207-2221. 